\title{
PIE DIABÉTICO. RECOMENDACIONES DE LA FEDERACIÓN INTERNACIONAL DE DIABETES 2017
}

\author{
DIABETIC FOOT. RECOMMENDATIONS OF THE \\ INTERNATIONAL DIABETES FEDERATION 2017
}

La diabetes mellitus y sus complicaciones se están convirtiendo rápidamente en la causa más significativa de morbimortalidad. Se predice que para el año 2040 habrá 642 millones de personas con diabetes en el mundo, con una incidencia de úlceras del pie de más del $25 \%$. Es por ello que debemos enfocarnos en la prevención de dichas lesiones más que en su tratamiento. Los índices de morbilidad de la diabetes son exageradamente altos, y los índices de mortalidad a cinco años después de la amputación de un miembro inferior, son apenas superados por la del cáncer de pulmón.

La hiperglucemia eleva el riesgo de aparición de complicaciones micro y macrovasculares, complicaciones como la retinopatía, enfermedad cardiovascular, nefropatía y neuropatía periférica, la cual es la principal causa de aparición de úlceras de los pies y lidera las amputaciones de miembros inferiores.

Es necesario un cambio urgente de paradigma para prevenir las lesiones del pie en los pacientes diabéticos. Las úlceras del pie usualmente preceden la mayoría de las amputaciones de los miembros inferiores. Más de la mitad de las úlceras se infectará y requerirá hospitalización, mientras que más del $20 \%$ resultará en una amputación.

Los problemas del pie son en efecto un problema global y no hay área en el mundo que no reporte el desarrollo de lesiones del pie como consecuencia de la neuropatía y/o enfermedad vascular periférica.

La prevalencia de una úlcera del pie activa varía desde el $1 \%$ en Europa y Estados Unidos hasta el $11 \%$ en los países africanos. Aunque hubo bastantes progresos en los últimos años respecto del tratamiento de las lesiones del pie, aún queda mucho por hacer dado que la mayoría de los adelantos se basa en el tratamiento de las complicaciones ya existentes y no en la prevención de las mismas.

El pie diabético es un problema médico, social y económico en todo el mundo. Sin embargo los reportes de ulceraciones y amputaciones varían considerablemente debido a diferencias de criterio de diagnóstico y tratamiento, como así también a la existencia de diferentes problemas económicos y de cuidados de Salud Pública en diferentes regiones.

En la mayoría de los países desarrollados la incidencia anual de úlceras del pie en pacientes con diabetes es de aproximadamente el $2 \%$. Es la causa más común de amputación no traumática; cerca del $1 \%$ de los pacientes con diabetes sufre una amputación de miembro inferior.

En los países en vías de desarrollo las úlceras del pie y las amputaciones son mucho más comunes. La pobreza, la falta de sanidad e higiene y el hábito de caminar descalzos son factores que impactan en el daño del pie de estos pacientes. En países de bajos ingresos, la falta de acceso a cuidados de salud adecuados junto con factores económicos y geográficos hacen que varios de estos pacientes no puedan alcanzar un tratamiento médico adecuado hasta que las lesiones presentan severos signos de infección y ya es tarde para medidas terapéuticas adecuadas, mucho menos preventivas.

En Latinoamérica las amputaciones son la causa más frecuente de admisión hospitalaria y las amputaciones por diabetes alcanzan un rango de 45 a $75 \%$, pudiendo encontrar hasta un $10 \%$ de pacientes con pie de riesgo en la población atendida en nivel primario, de acuerdo a los escasos estudios nacionales de Argentina, Brasil, Barbados y Cuba. Los sistemas de salud no están lo suficientemente organizados aún, la cobertura es incompleta y el copago no siempre es óptimo para la población total. De esta forma, las úlceras y amputaciones se convierten en un problema médico mayor con consecuencias sociales y económicas para el paciente, sus familias y el sistema de salud. El Estudio 
BRAZUPA del año 2016, realizado en 19 centros de Brasil con un total de 1.455 pacientes, es una de las últimas investigaciones estadísticas realizadas en Latinoamérica. Es indispensable la recolección de mayor cantidad de datos estadísticos en la región.

La prevalencia de neuropatía diabética periférica presenta variaciones entre el 16 y el $66 \%$. Debido a la falta de entrenamiento se ha estimado que menos de una tercera parte de los médicos reconoce los síntomas de neuropatía diabética periférica, aún cuando ésta es sintomática y debatan sobre ello con sus pacientes. La oportunidad de enfrentar la diabetes y la progresión de sus complicaciones está en diagnosticarlas y tratarlas en etapas tempranas.

El entendimiento y la comprensión del manejo del pie diabético faltan en gran parte de los profesionales integrantes del equipo de salud. Los cuidados del pie diabético han sido descriptos como "fragmentados y hechos al azar o irregularmente" dependiendo de los conocimientos del profesional que asista al paciente y de la disponibilidad de recursos. En muy pocos centros el pie diabético se trata con métodos sistemáticos y estandarizados. Es preciso contar con una guía de bolsillo para que los profesionales sepan realizar un examen clínico del pie basado en conocimientos completos para actuar preventivamente.

En el momento del diagnóstico de diabetes, así como en los chequeos regulares, el profesional debe advertir cualquier signo de alarma y evaluar la urgencia y el tratamiento temprano y preventivo del pie diabético de acuerdo a su categoría de riesgo.

A todo paciente con diabetes se le debe realizar un screening del pie para estratificarlo adecuadamente según el riesgo que tenga, lo cual incluye el examen clínico para realizar la prevención y el tratamiento. Es necesario el trabajo de un equipo de profesionales que incluya médicos diabetólogos, cirujanos vasculares, traumatólogos, infectólogos, enfermeros entrenados en el cuidado del pie, profesionales capacitados para la curación y cicatrización de heridas, podólogos, ortoprotesistas, kinesiólogos y expertos en educación diabetológica y cuidados domiciliarios; todo el equipo es indispensable para lograr buenos resultados y mejorar la calidad de vida del paciente.

En 2017 se publicaron las Guías de Recomendaciones de la Federación Internacional de Diabetes (IDF, sus siglas en inglés) cuyo objetivo es proteger el pie diabético desde el inicio y prevenir ulceracio- nes y amputaciones, para lo cual es preciso tomar medidas preventivas tempranas en el proceso de la enfermedad y en el tratamiento del pie desde la categoría de riesgo 1 y 2 , antes que se convierta en categoría 3 ("muy alto riesgo").

Cerca del $8 \%$ de los costos de pie diabético se utiliza en pie con categoría 3, por ello es necesario focalizar tempranamente el tratamiento de estos pacientes con el objetivo de prevenir úlceras y la progresión hacia la categoría de riesgo 3 . El presupuesto de salud de cada país no podrá sustentar la demanda necesaria para tratar las complicaciones del pie diabético, tales como úlceras y amputaciones, dado que esta enfermedad progresa incesantemente. Los conocimientos sobre el cuidado del pie diabético -basados en la prevención, educación y atención de un equipo multidisciplinario- pueden reducir las complicaciones del pie y las amputaciones en un $85 \%$. En resumen, necesitamos enfocar nuestros recursos hacia el tratamiento precoz en las categorías de riesgo y no focalizar en el tratamiento reactivo de las úlceras ya establecidas.

- Categoría de riesgo 0: sensibilidad plantar presente. Bajo riesgo.

- Categoría de riesgo 1: pérdida de la sensibilidad. Riesgo moderado.

- Categoría de riesgo 2: pérdida de la sensibilidad asociada a aumento de presiones o alteraciones en la circulación o deformidades estructurales del pie u onicomicosis. Riesgo alto.

- Categoría de riesgo 3: antecedentes de ulceraciones, amputaciones o fractura neuropática. Muy alto riesgo.

Es indispensable que las autoridades gubernamentales apliquen políticas en Salud Pública que promuevan la formación de equipos multidisciplinarios entrenados en el tratamiento del pie diabético, como así también obtener datos estadísticos fehacientes de las diferentes regiones del país para optimizar de este modo la atención de los pacientes, aún para aquellos que vivan en lugares alejados de los centros de salud.

Dra. Marta S. Calvagno Médica Especialista en Nutrición Prof. Adjunta de la Cátedra de Nutrición, Universidad de Buenos Aires, Ciudad Autónoma de Buenos Aires, Argentina 


\section{BIBLIOGRAFÍA}

International Diabetes Federation. IDF Diabetes Atlas, $7^{\text {th }}$ Ed. Brussels, Belgium: International Diabetes Federation, 2015. http://www.diabetesatlas.org.

- Singh N, Armstrong DG, Lipsky BA. Preventing foot ulcers in patients with diabetes. JAMA 2005 Jan 12; 293(2):217-28.

- Armstrong DG, Wrobel J, Robbins JM. Guest editorial: are diabetes-related wounds and amputations worse than cancer. Int Wound J 2007 Dec 1; 4(4):286-7.

- World Health Organization. Assessing national capacity for the prevention and control of noncommunicable diseases. Report of the 2015 global survey. Geneva: WHO. 2015.

- World Health Organization. Global status report on noncommunicable diseases 2014.

- Hu FB. Globalization of diabetes. Diabetes Care 2011 May 26; 34(6):1249.

- Wu SC, Driver VR, Wrobel JS, Armstrong DG. Foot ulcers in the diabetic patient, prevention and treatment. Vascular health and risk management 2007 Feb 1; 3(1):65.

- Boulton AJ. The diabetic foot: a global view. Diabetes/metabolism research and reviews 2000 Sep 1; 16(S1):S2-5.

- Boulton A. The diabetic foot: epidemiology, risk factors and the status of care. Diabetes Voice 2005 Nov; 50(S1):5-7.
- Boulton AJ, Vileikyte L, Ragnarson-Tennvall G, Apelqvist J.The global burden of diabetic foot disease. The Lancet 2005 Nov 18; 366(9498):1719-24.

- Bobircã F, Mihalache O, Georgescu D, Pãtraæcu T. The new prognostic-therapeutic index for diabetic foot surgery-extended analysis. Chirurgia 2016; 111:151-5.

- Lazzarini PA, Hurn SE, Fernando ME, Jen SD, Kuys SS, Kamp MC, Reed LF. Prevalence of foot disease and risk factors in general inpatient populations: a systematic review and meta-analysis. BMJ Open 2015 Nov 1; 5(11):e008544.

- Melmed S, Polonsky KS, Larsen PR, Kronenberg HM. Williams textbook of Endocrinology $13^{\text {th }}$ Edition 2016. Elsevier Inc.

- Cheung C, et al. The diabetic foot: a reconceptualization. Diabetic Foot Canada 2013; Vol 1, N 1, 11-12.

- International Diabetes Federation and International Working Group of the Diabetic Foot. Diabetes and foot care: time to act. Fourth Edition. IDF 2017.

- Resende KF. Predisposing factors for amputation of lower extremities in diabetic patients with foot ulceration in the state of Sergipe. J Vasc Bras 2006; 5 (2): 123-30

- Boulton AJM, Vileikyte L, Ragnarson-Tennvall G, Apelqvist J. The global burden of diabetic foot disease. Lancet 2005; 366:1719-1724. 